\title{
Natural Images Allow Universal Adversarial Attacks on Medical Image Classification Using Deep Neural Networks With Transfer Learning
}

\author{
Akinori Minagi \\ Kyushu Institute of Technology \\ Hokuto Hirano \\ Kyushu Institute of Technology \\ Kazuhiro Takemoto ( $\nabla$ takemoto@bio.kyutech.ac.jp ) \\ Kyushu Institute of Technology
}

\section{Research Article}

Keywords: deep neural networks, transfer learning, medical imaging, adversarial attacks, security and privacy

Posted Date: October 1st, 2021

DOI: https://doi.org/10.21203/rs.3.rs-757225/v2

License: (c) (i) This work is licensed under a Creative Commons Attribution 4.0 International License. Read Full License

Version of Record: A version of this preprint was published at Journal of Imaging on February 4th, 2022. See the published version at https://doi.org/10.3390/jimaging8020038. 


\title{
Natural images allow universal adversarial attacks on medical image classification using deep neural networks with transfer learning
}

\author{
Akinori Minagi ${ }^{1}$, Hokuto Hirano ${ }^{1}$, Kazuhiro Takemoto ${ }^{\text {* }}$ \\ 1) Department of Bioscience and Bioinformatics, Kyushu Institute of Technology, Iizuka, \\ Fukuoka 820-8502, Japan \\ *Corresponding author's e-mail: takemoto@bio.kyutech.ac.jp \\ Abstract
}

Background. Transfer learning from natural images is well used in deep neural networks (DNNs) for medical image classification to achieve computer-aided clinical diagnosis. Although the adversarial vulnerability of DNNs hinders practical applications owing to the high stakes of diagnosis, adversarial attacks are expected to be limited because training data - which are often required for adversarial attacks - are generally unavailable in terms of security and privacy preservation. Nevertheless, we hypothesized that adversarial attacks are also possible using natural images because pre-trained models do not change significantly after fine-tuning.

Methods. We considered three representative DNN-based medical image classification tasks (i.e., skin cancer, referable diabetic retinopathy, and pneumonia classifications) to investigate whether medical DNN models with transfer learning are vulnerable to universal adversarial perturbations (UAPs), generated using natural images.

Results. UAPs from natural images are useful for both non-targeted and targeted attacks. The performance of UAPs from natural images was significantly higher than that of random controls, although slightly lower than that of UAPs from training images. Vulnerability to UAPs from natural images was observed between different natural image datasets and between different model architectures.

Conclusion. The use of transfer learning causes a security hole, which decreases the reliability and safety of computer-based disease diagnosis. Model training from random initialization (without transfer learning) reduced the performance of UAPs from natural images; however, it did not completely avoid vulnerability to UAPs. The vulnerability of UAPs from natural images will become a remarkable security threat.

33 Keywords: deep neural networks, transfer learning, medical imaging, adversarial attacks, security and privacy 
36 Transfer learning from natural image datasets (e.g., the ImageNet dataset [1]) is a widely used technique in deep neural networks (DNNs) for image classification; in particular, it has been well applied to medical imaging [2]. Although the amount of medical image data is often limited, transfer learning enables the acquisition of highly accurate DNNs from such limited image data by fine-tuning existing model architectures (e.g., Inception V3 [3] and ResNet50 [4]), pre-trained using the ImageNet dataset with the image data. The transfer learning technique has been used for skin cancer classification based on photographic images [5], referable diabetic retinopathy classification based on optical coherence tomography (OCT) images of the retina [6], and pneumonia classification based on chest X-ray images [6]. The diagnostic performance of these DNNs is high and equivalent to that of healthcare professionals [7]. Thus, DNNs with transfer learning are beginning to be applied to medical image diagnosis to empower physicians and accelerate decision-making in clinical environments [2].

However, the practical application of DNNs to disease diagnosis may still be debatable owing to the existence of adversarial examples [8-10]; these are input images that are typically generated by adding specific, imperceptible perturbations to the original input images, leading to DNN misclassification. Given that diagnosing disease involves making high-stake decisions, the existence of adversarial examples is a security concern [11]. Adversarial examples probably cause misdiagnosis and various social disturbances [12], as well as limiting deep learning applications in safety- and security-critical environments [13]. Therefore, it is also important to evaluate the reliability and safety of DNNs against adversarial attacks in medical imaging.

Many previous studies have demonstrated that DNN models are vulnerable to inputdependent adversarial attacks (i.e., an individual adversarial perturbation is used such that each input image is misclassified) in skin cancer [12] and pneumonia classifications [14]. More importantly, a previous study [15] showed that a single small image agnostic perturbation, called universal adversarial perturbation (UAP) $[16,17]$, can induce DNN failure in most tasks and is also a security threat to DNN-based medical image classifications. UAP-based attacks are more realistic because they can be more easily implemented by adversaries in real-world environments and are practicable, with a lower computational cost [16].

A simple solution to avoid adversarial attacks is to render training data and any other similar domain-specific data (e.g., medical images in the case of medical image classification) publicly unavailable because various methods of adversarial attacks [8-10] (from attack methods that assume access to DNN model weights to those that do not) generally assume the use of such data to generate adversarial perturbations. Given that the data availability of medical images is generally limited in terms of security and privacy preservation [11], adversarial attacks on DNN-based medical image classifications seem to be limited. However, we doubt this prediction because of the properties of transfer learning 75 for medical imaging [18]. Specifically, transfer learning considers that model weights pre76 trained with the ImageNet dataset (natural images) are fine-tuned with medical images; 77 however, fine-tuned DNN models for medical imaging are known to be almost similar to 
the original pre-trained DNN models, despite the fine-tuning process. Additionally, larger DNN models do not change through training. It seems that DNN models obtained by finetuning well-used model architectures (e.g., Inception V3 and ResNet50) with medical images show almost similar reactions to both medical and natural images.

Thus, we developed and tested the hypothesis that adversarial perturbations against finetuned DNN models are generatable using not only training data (medical images) but also natural images (e.g., the ImageNet dataset). Following our previous study [15], we considered representative medical image classifications (skin cancer classification based on photographic images [5], referable diabetic retinopathy classification based on OCT images [6], and pneumonia classification based on chest X-ray images [6]) and investigated the vulnerability of fine-tuned DNN models with several architectures to adversarial perturbations generated using natural images. In this study, we focused on universal adversarial attacks $[16,17]$ rather than input-dependent adversarial attacks. This is because input-dependent adversarial attacks are less effective; in particular, it is costly to determine the medical images that result in misclassification from an adversarial perturbation generated using a natural image. In contrast, UAPs (generated using natural images) can be used for any medical image because they are image agnostic. To evaluate the effects of transfer learning on vulnerability to UAPs, we also considered the DNN model architecture training from random initialization.

\section{Methods}

\section{Medical image datasets and models}

We used the medical image datasets and DNN models previously described in [15] (see also github.com/hkthirano/MedicalAI-UAP). A brief description is provided below:

The skin lesion images for skin cancer classification comprised 7,000 training images and 3,015 test images that were classified into seven classes: melanoma (MEL), melanocytic nevus (NV), basal cell carcinoma (BCC), actinic keratosis/Bowens disease (intraepithelial carcinoma; AKIEC), benign keratosis (solar lentigo/seborrheic keratosis/lichen planus-like keratosis; BKL), dermatofibroma (DF), and vascular lesions (VASC). The OCT images for referable diabetic retinopathy classification consisted of 7,840 training and 3,360 test images that were classified into four classes: choroidal neovascularization with neovascular membrane and associated subretinal fluid $(\mathrm{CNV})$, diabetic macular edema with retinal-thickening-associated intraretinal fluid (DME), multiple drusen present in early age-related macular degeneration (DRUSEN), and normal retina with preserved foveal contour and absence of any retinal fluid/edema (NM). The chest X-ray images for pneumonia classification comprised 1,800 training and 540 test images that were classified into binary classes: no pneumonia (NORMAL) or viral or bacterial pneumonia (PNEUMONIA). Note that the OCT and chest X-ray image datasets were class-balanced but the skin lesion image dataset was not.

The Inception V3 architecture [3] was mainly considered, following previous studies [5, 6]. To evaluate the effect of model architecture on vulnerability to UAPs, we also used the VGG16 [19] and ResNet50 [4] architectures. These DNN model architectures pre-trained using the ImageNet dataset were fine-tuned with the training images in a medical image 
120 dataset, using the learning rate schedule and data augmentation. We also trained the 121 Inception V3 models with the training images in a medical image dataset from random 122 initialization to evaluate the effects of transfer learning on the vulnerability to UAPs from 123 natural images. The training conditions in this case (e.g., the learning rate schedule, 124 condition of data augmentation, and number of epochs) were identical to those in the case of transfer learning, except for random initialization. \\ 126 Universal adversarial perturbations and natural images}

127 Following our previous study[15], simple iterative algorithms [16, 17] were used to 128 generate UAPs. We considered both non-targeted attacks, which cause misclassification 129 (i.e., a task failure resulting in an input image being assigned an incorrect class), and 130 targeted attacks, which caused the DNN to classify an input image into a specific class. for 131 the non-targeted and targeted attacks, respectively. The Adversarial Robustness 360 132 Toolbox (ART) [20] (version 1.0; github.com/Trusted-AI/adversarial-robustness-toolbox) 133 was used. For targeted UAPs, we used our proposed method [17] (see also 134 github.com/hkthirano/targeted_UAP_CIFAR10), which was implemented by modifying 135 the nontargeted UAP algorithm [16].

136 The algorithms consider a classifier and generate UPAs $\boldsymbol{\rho}$ from an input image set $\boldsymbol{X}$, 137 under the constraint that the $L_{p}$ norm of the perturbation $\|\rho\|_{p} \leq \xi$, for a small $\xi$ value. 138 The algorithms start with $\boldsymbol{\rho}=\mathbf{0}$ (no perturbation) and iteratively update $\boldsymbol{\rho}$ by additively 139 obtaining an adversarial perturbation for an input image $\boldsymbol{x}$, which is randomly selected 140 from $\boldsymbol{X}$ without replacement via the fast gradient sign method [8] with the attack strength 141 parameter $\epsilon$. These iterative updates continue until the number of iterations reaches a 142 maximum $i_{\max }$.

143 Using these algorithms, UAPs against a DNN model for medical image classification were 144 generated using natural images. We considered the training images in the ImageNet dataset 145 because the DNN models were pre-trained using the ImageNet dataset. The ImageNet 146 training set was downloaded from www.image-net.org/download.php on June 17, 2020. 147 Moreover, we also considered the Open Images dataset (V6), a different dataset of natural 148 images, to evaluate the dataset dependency on the performance of UAPs. The dataset was 149 downloaded from storage.googleapis.com/openimages/web/download.html on November 150 22, 2020. For each dataset, 100,000 randomly selected images were used to generate the 151 UAPs. The images were gray-transformed when generating UAPs against the DNN models 152 for referable diabetic retinopathy and pneumonia classifications.

153 For skin lesion and chest X-ray image classifications, the parameters $\epsilon$ and $p$ were set to 1540.0005 and 2, respectively. For the OCT image classification, $\epsilon$ and $p$ were set to 0.0013 155 and $\infty$, respectively. However, different $\epsilon$ was considered for the Inception V3 models 156 trained from random initialization. When generating UAPs using training images, $\epsilon$ was $1570.0044,0.0036$, and 0.0066 for the skin lesion, OCT, and chest X-ray image classifications, 158 respectively. When generating UAPs using natural images, $\epsilon$ was $0.0050,0.0020$, and 1590.0026 for the skin lesion, OCT, and chest X-ray image classifications, respectively. The 160 parameters $\epsilon$ and $p$ were selected using a grid search to maximize the performance of 161 the UAPs (see below) for input images. The parameter $i_{\max }$ was set to 1 . The parameter 
$\xi$ was set based on the ratio $\zeta$ of the $L_{p}$ norm of the UAP to the average $L_{p}$ norm of an

163 image in the dataset (see [15] for the actual values of the average $L_{p}$ norms).

164 We also obtained the UAPs generated using the training images in a medical dataset from 165 our previous study [15] to compare the performances of the UAPs between the training 166 images and natural images. Random vectors (random UAPs) were sampled uniformly from 167 the sphere of a specified radius to compare the performances of the generated UAPs with 168 those of the random controls [16].

\section{Evaluating the performance of UAPs}

170 Evaluating the performance of UAPs was based on the procedures established in our 171 previous study [15]. The fooling rate $R_{f}$ and targeted attack success rate $R_{s}$ was used to 172 evaluate the performance of a nontargeted UAP $\left(\boldsymbol{\rho}_{\mathrm{nt}}\right)$ and targeted $\operatorname{UAP}\left(\boldsymbol{\rho}_{\mathrm{t}}\right) . R_{f}=$ $173|\boldsymbol{X}|^{-1} \sum_{\boldsymbol{x} \in X} \mathbb{I}\left(C(\boldsymbol{x}) \neq C\left(\boldsymbol{x}+\boldsymbol{\rho}_{\mathrm{nt}}\right)\right)$, where $C(\boldsymbol{x})$ be an output (class or label) of a 174 classifier (DNN) for an input image $\boldsymbol{x}$ in an image set $\boldsymbol{X}$. The function $\mathbb{I}(A)$ returns 1 175 if the condition $A$ is true and 0 otherwise. $R_{f}$ indicates the fraction of adversarial images 176 from which the labels predicted are inconsistent with the labels predicted from clean 177 images to all images in the set. $R_{S}=|\boldsymbol{X}|^{-1} \sum_{\boldsymbol{x} \in \boldsymbol{X}} \mathbb{I}\left(C\left(\boldsymbol{x}+\boldsymbol{\rho}_{\mathrm{t}}\right)=y\right)$ : the proportion of 178 adversarial images classified into the target class $y$ to all images in set $\boldsymbol{X}$. Note that $R_{S}$ 179 has a baseline, $R_{S}$ observed without UAPs. As mentioned in our previous study [15], the 180 label composition of the image data and prediction performance of DNNs both affect the 181 baseline. The $R_{S}$ baselines of UAPs targeted to a specified class were $\sim 25 \%$ and $\sim 50 \%$, 182 respectively, for the OCT and chest X-ray image datasets. For the skin lesion dataset, the $183 R_{s}$ baselines of UAPs targeted to MEL and NV were $\sim 10 \%$ and $\sim 65 \%$, respectively. $R_{f}$ 184 and $R_{S}$ were computed using test images from the medical image dataset. The confusion 185 matrices on test images from the medical image dataset were also obtained to evaluate the 186 change in prediction owing to the UAPs for each class. The rows and columns in the 187 matrices represent the true and predicted classes, respectively. The confusion matrices were 188 row-normalized to account for imbalanced datasets.

\section{Results}

190 Natural images allow nontargeted universal adversarial attacks on medical 191 image classification

192 We first consider the Inception V3 models as it was used by previous studies on DNN193 based medical imaging [5, 6] and evaluated whether nontargeted UAPs against the DNN 194 models for medical image classification are generatable using natural images (Fig. 1). The 195 performance of the UAPs generated using the natural images was less effective than that 196 of the UAPs generated in the training images in the medical image dataset; specifically, the 197 UAPs from the training images achieved a higher fooling rate $R_{f}$, with a smaller 198 perturbation magnitude $\zeta$, compared to the UAPs from the natural images. However, $R_{f}$ 199 of the UAPs generated using the natural images was significantly higher than that of 200 random UAPs; moreover, they also increased rapidly with $\zeta$ and reached a high $R_{f}$, 201 despite a low $\zeta$. Specifically, $R_{f} \sim 80 \%$ and $\sim 50 \%$ were achieved at $\zeta=4 \%$ for the skin 
lesion (Fig. 1a) and chest X-ray image classifications (Fig. 1c), respectively. $R_{f}$ was $40-$ $60 \%$ at $\zeta=8 \%$ for the OCT image classification (Fig. 1b). These UAPs were almost imperceptible. As a representative example, clean images and their adversarial examples owing to the UAPs from the ImageNet dataset are shown in Fig. 2. These results indicate that small UAPs from natural images also cause misclassification of DNN-based medical image classifications. We also found that the performance of UAPs from natural images

208 has no strong dataset dependency because $R_{f}$ of the UAPs from the Open Images dataset 209 was almost similar to those of the UAPs generated using the ImageNet dataset, although 210 small differences in $R_{f}$ were observed, i.e., $\sim 40 \%$ and $\sim 60 \%$ for the Open images and 211 ImageNet datasets, respectively.

212 For the ResNet50 and VGG16 models, $R_{f}$ of the UAPs from the natural images was also 213 significantly higher than that of the random control (Fig. 3), although it was less than that 214 of the UAPs from the training images. However, $R_{f}$ at the same $\zeta$ was different between the model architectures, except for the chest X-ray image classification. For the skin lesion image classification (Fig. 3a), $R_{f}$ of the UAPs with $\zeta=4 \%$ was approximately $80 \%$ for the Inception V3 model, whereas it was lower for the ResNet50 and VGG16 models.

218 Specifically, $R_{f}$ against the ResNet50 model and the VGG model were $\sim 70 \%$ and $30-$ $21950 \%$, respectively. For the OCT image classification (Fig. 3b), a slightly higher $R_{f}(60-$ $22070 \%$ ) of the UAPs against ResNet50 and VGG16 models with $\zeta=8 \%$ was observed, compared to the Inception V3 model $(40-60 \%)$. For the chest X-ray image classification (Fig. 3c), $R_{f}$ of the UAPs with $\zeta=4 \%$ from the natural images was $\sim 50 \%$, independent of the model architecture.

As expected from the observed difference in $R_{f}$ between the UAPs from training images, and those generated from natural images, those from the natural images were visually different to those from the training images, for the same $\zeta$. Figure 4 shows the UAPs generated using the training, ImageNet, and Open Images datasets against Inception V3 models. Moreover, the confusion matrices on the test images also showed a different tendency of misclassification of the DNN models due to UAPs between the UAPs from the natural images and those from the training images, although the confusion matrix patterns are similar in that the DNN models classify most images into several specific classes (i.e., dominant classes) due to UAPs. Figure 5 shows the Inception V3 model. For the skin lesion image classification, the dominant classes were MEL and BLK when using the UAPs from the training images; however, the dominant class was only MEL when using the UAPs from the natural images (both the ImageNet and Open Images datasets). For the OCT image classification, the dominant class was $\mathrm{CNV}$ in the case of the UAPs from the training images; however, it was DRUSEN and NM in the case of the UAPs from the ImageNet dataset and in the case of the UAPs from the Open Images dataset. For the chest X-ray image classification, the DNN model incorrectly predicted the true labels because of the UAPs from the training images; however, it classified most images into NORMAL because of the UAPs from the natural images (both the ImageNet and Open Images datasets), indicating that $R_{f}$ saturated at $\sim 50 \%$ (Fig. $1 \mathrm{c}$ ).

243 The dominant classes might differ according to the model architectures and natural image datasets, except for the chest X-ray image classification. For the skin lesion classification, 
the dominant class was BKL for the UAPs from both the ImageNet and Open Images datasets against the VGG16 model and for the UAP from the Open Images dataset against ResNet50, whereas it was MEL for the UAPs from the ImageNet dataset against the ResNet50 model (Additional file 1: Fig. S1). For the OCT image classification, the dominant classes of the UAPs from both the ImageNet and Open Images datasets were DRUSEN for ResNet50; however, they were CNVs for the VGG16 model (Additional file 1: Fig. S2). For the chest X-ray image classification, the dominant classes were NORMAL, independent of the model architectures and natural image datasets (Additional file 1: Fig. S3).

Natural images allow targeted universal adversarial attacks on medical image classification

We also investigated whether the targeted UAPs were generatable using natural images. Following our previous study [15], we considered targeted attacks to be the most significant case and the control in each medical image dataset. The most significant cases correspond to MEL, CNV, and PNEUMONIA in the skin lesion, OCT, and chest X-ray image datasets, respectively. The controls correspond to NV, NM, and NORMAL in the skin lesion, OCT, and chest X-ray image datasets, respectively. Table 1 presents the targeted attack success rate $R_{S}$ of the UAPs against DNN-based medical image classification. The UAPs were very small and almost imperceptible because $\zeta=4 \%$ in the skin lesion and chest X-ray image classifications and $\zeta=8 \%$ in the OCT image classification, as in the case of nontargeted UAPs (see Fig. 2). However, overall, the values of $R_{S}(>90 \%)$ of the UAPs from both the ImageNet and Open Images datasets were significantly higher than those of random UAPs; moreover, they were almost similar to those of the UAPs from the training images in the medical image dataset. This tendency was independent of the model architectures. However, low $R_{S}$ was observed in a small number of cases. $R_{S}$ of the UAPs from the ImageNet and Open Images targeted to MEL were $\sim 10 \%$, which were almost similar to the random control for the ResNet50 model, whereas they were $\sim 95 \%$ for the Inception V3 and ResNet50 models. $R_{S}$ of the UAPs from the ImageNet and Open Images targeted to $\mathrm{CNV}$ were $35-50 \%$, which were higher than random controls for the ResNet50 model, whereas they were $\sim 100 \%$ for Inception V3 and VGG16 models. $R_{S}$ of the UAPs from the ImageNet and Open Images targeted to PNEUMONIA were $60-80 \%$, which was higher than that of random controls, whereas that of the UAPs from the training images was $\sim 100 \%$.

278 As representative examples, Figure 6 shows the UAPs generated using several image 279 datasets for targeted attacks on MEL, CNV, and NORMAL against Inception V3 models. 280 These UAPs showed $R_{S} \sim 100 \%$; however, the UAPs from natural images were visually

\section{Effect of transfer learning on the vulnerability of the UAPs from natural images}

283 It is predicted that transfer learning from natural images (the ImageNet dataset, in particular) causes the observed vulnerability of the UAPs from natural images to DNNbased medical image classification. To test this more deeply, we considered the Inception V3 models, which are widely used in medical image classification $[5,6]$, trained with the 
training images in each medical image dataset from random initialization. The test accuracies of the models were $79.2 \%, 95.3 \%$, and $97.8 \%$ for the skin lesion, OCT, and chest X-ray image datasets, respectively. The accuracies of the models trained from random initialization were almost similar to those $(95.5 \%$ and $97.6 \%$, respectively [15]) of the models trained from transfer learning for the OCT and chest X-ray image datasets; however, the accuracy from random initialization was slightly lower than that $(87.7 \%$ [15]) from transfer learning.

294 We evaluated the vulnerability of nontargeted UAPs against these Inception V3 models 295 (Table 2) and found that the UAPs from natural images were partly less effective for fooling 296 the DNN-based medical image classifications. For the skin lesion image classification, the $297 R_{f}$ of the UAP from the ImageNet dataset was only $\sim 50 \%$, despite a larger $\zeta(\zeta=8 \%$, 298 i.e., two times larger than the case show in Fig. 3a), whereas $R_{f}$ of the UAP from the 299 training images was $\sim 90 \%$. For the chest X-ray image classification, $R_{f}$ of the UAP from 300 the ImageNet dataset was only $\sim 20 \%$ despite a larger $\zeta(\zeta=8 \%$, i.e., two times larger than the case shown in Fig. 3c), whereas $R_{f}$ of the UAP from the training images was $\sim 45 \%$. The results indicate that model training from random initialization reduces the performance of UAPs from natural images. However, the vulnerability of UAPs from natural images is not completely avoided because of random initialization. $R_{f}$ of the UAPs from the ImageNet dataset was still larger than that of random UPAs, $R_{f}$ was almost similar between the UAPs from the ImageNet dataset and the UAPs from the training images for the OCT image classification, although $\zeta=16 \%$, i.e., two times larger than the case shown in Fig. 3b).

\section{Discussion}

310 We hypothesized that UAPs against DNN models with transfer learning are also 311 generatable using natural images because pre-trained models do not change significantly 312 after fine-tuning. We further demonstrate that fine-tuned models for medical image 313 classification are vulnerable to both non-targeted and targeted UAPs generated using 314 natural images (Fig. 1 and Table 1). Vulnerability to both non-targeted and targeted UAPs 315 from natural images was confirmed in several model architectures. Vulnerability to UAPs 316 may be a universal feature in DNNs. Given the fact that the DNN models for medical image classification with transfer learning from the ImageNet dataset are vulnerable not only to UAPs from the ImageNet dataset but also to UAPs from the Open Images datasets, this vulnerability to the UAPs may be independent of natural image datasets, indicating that

320 UAPs against DNN models with transfer learning are generatable using any publicly321 available natural images. This may be a novel security threat to DNN-based medical image diagnosis, in particular, it indicates that almost imperceptible UAPs are generatable without training medical data and any other similar medical data (no matter how much such data are kept a secret). Unlike the prediction that adversarial attacks on DNN-based medical image classifications are difficult because data availability of medical images is generally limited in terms of security and privacy preservation, the results indicate that a DNN-based medical image diagnosis is easier to deceive. Adversaries can not only result in failed DNN-based medical image diagnoses but can also control DNN-based medical image diagnoses, even if they never access such medical data. 
330 The UAPs from natural images seem to be different from those from training images (Figs.

3314 and 6) and the characteristics (e.g., $R_{f}$, dominant classes, and $R_{s}$ ) of the UAPs from

332 natural images were partly different from those of the UAPs from training images. This

333 may be because of the difference in the composition of the predicted labels between the

334 training images and natural images (Additional file 1: Tables S1 - S3). For chest X-ray

335 image classification, for example, $\sim 80 \%$ of both the ImageNet and Open Images datasets

336 were classified as PNEUMONIA regardless of model architecture (Additional file 1: Table

337 S3), whereas the training images were almost class-balanced. As the nontargeted attack

338 algorithm [16] considers maximizing $R_{f}$, a large $R_{f}$ is achieved when images with such

339 an abundant label are misclassified. On the other hand, misclassifying images with a less

340 abundant label has little advantage for maximizing $R_{f}$. The performance of nontargeted

341 UAPs is less effective (images with less abundant labels are difficult-to-fool) and less-

342 abundant labels tend to correspond to dominant classes when the predicted labels of natural

343 images are imbalanced. For the chest X-ray image classification, the dominant class of the

344 UAPs from natural images was NORMAL (Fig. 5); as a result, $R_{f}$ was saturated at $\sim 50 \%$

345 (Fig. 1c). The tendency of the dominant classes to correspond to the less-abundant

346 predicted labels (see Tables S1 and S2) was also observed for the skin lesion and OCT

347 image classification (Fig. 5). The imbalanced predicted labels of the natural images also

348 affect the performance of the targeted UAPs. As the targeted attack algorithm [21]

349 considers maximizing $R_{S}$, a large $R_{S}$ will have already been achieved for targeted attacks

350 to an abundant label in a dataset. Thus, UAPs are rarely updated in the iterative algorithm;

351 as a result, $R_{s}$ rarely increases. The targeted attacks to NM and PNEUMONIA, which are

352 the abundant labels in the dataset (Additional file 1: Tables S2 and S3), were less effective

353 for the OCT and chest X-ray image classifications, respectively (Table 2). The performance

354 of UAPs from natural images may increase by controlling the composition of the predicted

355 labels of the natural images (e.g., using data augmentation).

356 This study showed that the UAPs were generatable without training data. In this context,

357 UAPs from natural images are regarded as black-box attacks. However, UAPs are not

358 complete black-box attacks because they assume a white-box condition: model parameters

359 (e.g., the gradient of the loss function) are accessible. This was because the well-used UAP

360 algorithms [16, 17], which we also used, are limited to the white-box condition. However,

361 this limitation poses few problems for adversaries. As represented by COVID-Net [22], a

362 deep convolutional neural network design intended to detect COVID-19 cases from chest

363 X-ray images, DNN models are often developed as open-source projects by expecting that

364 both researchers and citizens data scientists will accelerate the development of highly

365 accurate yet practical deep learning solutions. Moreover, collaboration among multiple

366 institutions is required to develop DNN models with high diagnostic performance [23] and

367 the distribution of deep learning models has been proposed as an effective alternative to

368 sharing patient data. Even if model architectures and weights are publicly unavailable and

369 the loss gradient is not accessible, they may be estimated [24] because medical DNNs are

370 often developed by fine-tuning existing DNNs, such as VGG, ResNet, and Inception, pre-

371 trained using the ImageNet dataset, as this study considered. Furthermore, DNNs are aimed

372 at real-world usage (e.g., automated support for clinical diagnosis). The assumption that

373 adversaries cannot access DNN models may be unrealistic from these reasons.

374 Nevertheless, our findings may also be useful for developing black-box attack methods 
that estimate adversarial perturbations using only model outputs (e.g., confidence scores).

376 Several methods for black-box attacks have been proposed [25-27]. Although they are 377 limited to input-dependent adversarial attacks, universal adversarial attacks may be 378 possible under the black-box condition because CNNs are sensitive to the directions of the 379 Fourier basis functions [28]. However, these methods assume the use of domain-specific 380 data (e.g., medical images in the case of medical image classification) that are not included 381 in the training data. Our study indicates that this assumption is not required. Adversaries 382 may be able to perform black-box attacks more easily than previously thought, simply by 383 using natural images instead of domain-specific images.

384 A solution for avoiding the vulnerability of UAPs from natural images is to train DNN 385 models from random initialization (i.e., without pretrained weights). The performance of 386 UAPs from natural images was overall lower in the DNN model trained with random 387 initialization (Table 2), compared to the DNN models with transfer learning. This might be 388 because the model weights were different from the pretrained weights from the natural 389 images. However, training from random initialization does not completely prevent the 390 vulnerability of UAPs from natural images. As shown in Table 2, the performance of the 391 UAPs was still higher than that of random controls; moreover, it was almost similar to that 392 of UAPs from training images in some cases (e.g., the OCT image classification). In 393 addition, the trade-offs with prediction performance need to be considered. Because 394 transfer learning contributes to faster convergence [18], prediction performance may 395 decrease when training DNN models from random initialization, compared to transfer 396 learning, when considering the same number of training steps (epochs): this solution may 397 be unrealistic in terms of the practical desire to achieve high prediction performance with 398 a lower computational cost.

399 Adversarial defenses [29] also need to be considered to reduce vulnerability to UAPs. 400 Recent developments in adversarial defenses [30-33] are remarkable; however, 401 comprehensive comparative evaluations [34, 35] have shown that promising defense 402 methods are less effective than reported. A cat-and-mouse game is defending against 403 adversarial attacks [12]. It may be difficult to completely avoid security concerns caused 404 by adversarial attacks. The development and operation of secure, privacy-preserving, and 405 federated DNNs are needed in medical imaging [11].

\section{Conclusion}

407 Our study showed that natural images allow universal adversarial attacks on medical image 408 classification using deep neural networks with transfer learning. It has been expected that 409 adversarial attacks are limited because medical images used for training are generally 410 unavailable; however, existing algorithms can generate UAPs using natural images instead 411 of training images. Transfer learning from natural images is a widely used DNN-based 412 medical image classification because the amount of medical image data is often limited. 413 However, the use of transfer learning causes a security hole, thereby reducing the reliability 414 and safety of computer-based disease diagnosis. Our findings demonstrate a novel 415 vulnerability of DNNs to adversarial attacks and may help to increase the security of 416 DNNs; in particular, they are useful for designing the operation strategy of medical DNNs. 


\section{List of abbreviations}

418 AKIEC: actinic keratosis/Bowens disease (intraepithelial carcinoma)

419 BCC: basal cell carcinoma

420 BKL: benign keratosis (solar lentigo/seborrheic keratosis/lichen planus-like keratosis)

421 CNV: neovascular membrane and associated subretinal fluid

422 DF: dermatofibroma

423 DME: diabetic macular edema with retinal-thickening-associated intraretinal fluid

424 DNN: deep neural network

425 DRUSEN: multiple drusen present in early age-related macular degeneration

426 MEL: melanoma

427 NM: normal retina with preserved foveal contour and absence of retinal fluid/edema

428 NV: melanocytic nevus

429 OCT: optical coherence tomography

$430 \quad$ ResNet: residual network

431 UAP: universal adversarial perturbation

432 VASC: vascular lesion

433 VGG: visual geometry group

434 Declarations

435 Ethics approval and consent to participate

436 Not applicable.

437 Consent for publication

438 Not applicable.

439 Availability of data and material

440 All data generated and analyzed during this study are included in this published article 441 and its supplementary information files. The code and data used in this study are

442 available from our GitHub repository: github.com/kztakemoto/Natural_UAP. 


\section{Competing interests}

444 The authors declare that they have no competing interests.

$445 \quad$ Funding

446 This research was funded by JSPS KAKENHI (grant number 21H03545).

447 Authors'contributions

448 AM and KT conceived and designed the study. AM and HH prepared the data and model. 449 AM and KT coded and performed the experimental evaluations. AM and KT interpreted 450 the results. KT wrote the manuscript. All authors have approved the final manuscript for 451 publication.

452 Acknowledgments

453 We would like to thank Editage (www.editage.jp) for the English language editing. 454 


\section{References}

456 1. Russakovsky O, Deng J, Su H, Krause J, Satheesh S, Ma S, et al. ImageNet large scale 457 visual recognition challenge. Int J Comput Vis. 2015.

458 2. Litjens G, Kooi T, Bejnordi BE, Setio AAA, Ciompi F, Ghafoorian M, et al. A survey 459 on deep learning in medical image analysis. Med Image Anal. 2017;42 December 460 2012:60-88. doi:10.1016/j.media.2017.07.005.

461 3. Szegedy C, Vanhoucke V, Ioffe S, Shlens J, Wojna Z. Rethinking the Inception architecture for computer vision. In: 2016 IEEE Conference on Computer Vision and 463 Pattern Recognition (CVPR). IEEE; 2016. p. 2818-26. doi:10.1109/CVPR.2016.308.

464 4. He K, Zhang X, Ren S, Sun J. Deep residual learning for image recognition. In: 2016 465 IEEE Conference on Computer Vision and Pattern Recognition (CVPR). IEEE; 2016. p. 466 770-8. doi:10.1109/CVPR.2016.90.

467 5. Esteva A, Kuprel B, Novoa RA, Ko J, Swetter SM, Blau HM, et al. Dermatologist468 level classification of skin cancer with deep neural networks. Nature. 2017;542:115-8. 469 doi:10.1038/nature21056.

470 6. Kermany DS, Goldbaum M, Cai W, Valentim CCS, Liang H, Baxter SL, et al.

471 Identifying medical diagnoses and treatable diseases by image-based deep learning. Cell.

472 2018;172:1122-1131.e9. doi:10.1016/j.cell.2018.02.010.

473 7. Liu X, Faes L, Kale AU, Wagner SK, Fu DJ, Bruynseels A, et al. A comparison of 474 deep learning performance against health-care professionals in detecting diseases from medical imaging: a systematic review and meta-analysis. Lancet Digit Heal. 2019;1:e271-97. doi:10.1016/S2589-7500(19)30123-2.

8. Goodfellow IJ, Shlens J, Szegedy C. Explaining and harnessing adversarial examples. Int Conf Learn Represent. 2015. http://arxiv.org/abs/1412.6572.

479 9. Yuan X, He P, Zhu Q, Li X. Adversarial examples: attacks and defenses for deep 480 learning. IEEE Trans Neural Networks Learn Syst. 2019;30:2805-24.

481 doi:10.1109/TNNLS.2018.2886017.

482 10. Ortiz-Jimenez G, Modas A, Moosavi-Dezfooli S-M, Frossard P. Optimism in the face 483 of adversity: Understanding and improving deep learning through adversarial robustness. 484 2020. http://arxiv.org/abs/2010.09624.

485 11. Kaissis GA, Makowski MR, Rückert D, Braren RF. Secure, privacy-preserving and 486 federated machine learning in medical imaging. Nat Mach Intell. 2020;2:305-11. 487 doi:10.1038/s42256-020-0186-1.

488 12. Finlayson SG, Bowers JD, Ito J, Zittrain JL, Beam AL, Kohane IS. Adversarial 489 attacks on medical machine learning. Science. 2019;363:1287-9.

490 doi:10.1126/science.aaw4399. 
491 13. Matyasko A, Chau L-P. Improved network robustness with adversary critic. Proc

492 32nd Int Conf Neural Inf Process Syst. 2018;:10601-10610.

493 http://arxiv.org/abs/1810.12576.

494 14. Asgari Taghanaki S, Das A, Hamarneh G. Vulnerability Analysis of Chest X-Ray 495 Image Classification Against Adversarial Attacks. In: Understanding and Interpreting 496 Machine Learning in Medical Image Computing Applications. 2018. p. 87-94.

497 doi:10.1007/978-3-030-02628-8_10.

498 15. Hirano H, Minagi A, Takemoto K. Universal adversarial attacks on deep neural 499 networks for medical image classification. BMC Med Imaging. 2021;21:9.

500 doi:10.1186/s12880-020-00530-y.

501 16. Moosavi-Dezfooli SM, Fawzi A, Fawzi O, Frossard P. Universal adversarial 502 perturbations. Proc - 30th IEEE Conf Comput Vis Pattern Recognition, CVPR 2017. 503 2017;2017-Janua:86-94.

504 17. Hirano H, Takemoto K. Simple iterative method for generating targeted universal 505 adversarial perturbations. Algorithms. 2020;13:268. doi:10.3390/a13110268.

506 18. Raghu M, Zhang C, Kleinberg J, Bengio S. Transfusion: Understanding Transfer

507 Learning for Medical Imaging. In: Wallach H, Larochelle H, Beygelzimer A,

508 d¥textquotesingle Alché-Buc F, Fox E, Garnett R, editors. Advances in Neural

509 Information Processing Systems 32. Curran Associates, Inc.; 2019. p. 3347-57.

510 http://papers.nips.cc/paper/8596-transfusion-understanding-transfer-learning-for-medical-

511 imaging.pdf.

512 19. Simonyan K, Zisserman A. Very deep convolutional networks for large-scale image 513 recognition. In: 3rd International Conference on Learning Representations, ICLR 2015 514 Conference Track Proceedings. 2015.

515 20. Nicolae M-I, Sinn M, Tran MN, Buesser B, Rawat A, Wistuba M, et al. Adversarial 516 Robustness Toolbox v1.0.0. 2018. http://arxiv.org/abs/1807.01069.

517 21. Hirano H, Takemoto K. Simple iterative method for generating targeted universal

518 adversarial perturbations. In: Proceedings of 25th International Symposium on Artificial

519 Life and Robotics. 2020. p. 426-30. http://arxiv.org/abs/1911.06502.

520 22. Wang L, Lin ZQ, Wong A. COVID-Net: a tailored deep convolutional neural 521 network design for detection of COVID-19 cases from chest X-ray images. Sci Rep.

522 2020;10:19549. doi:10.1038/s41598-020-76550-z.

523 23. Chang K, Balachandar N, Lam C, Yi D, Brown J, Beers A, et al. Distributed deep 524 learning networks among institutions for medical imaging. J Am Med Informatics Assoc. $525 \quad 2018 ; 25: 945-54$.

526 24. Bortsova G, González-Gonzalo C, Wetstein SC, Dubost F, Katramados I, Hogeweg 527 L, et al. Adversarial attack vulnerability of medical image analysis systems: Unexplored 528 factors. Med Image Anal. 2021;73:102141. doi:10.1016/j.media.2021.102141. 

box adversarial attacks via genetic algorithm. Comput Secur. 2019;85:89-106. doi:10.1016/j.cose.2019.04.014. adversarial attacks. Proc 36th Int Conf Mach Learn. 2019;:2484-93. http://arxiv.org/abs/1905.07121.

27. Co KT, Muñoz-González L, de Maupeou S, Lupu EC. Procedural noise adversarial examples for black-box attacks on deep convolutional networks. In: Proceedings of the 2019 ACM SIGSAC Conference on Computer and Communications Security. New York, NY, USA: ACM; 2019. p. 275-89. doi:10.1145/3319535.3345660.

539 28. Tsuzuku Y, Sato I. On the structural sensitivity of deep convolutional networks to the directions of Fourier basis functions. In: IEEE Conference on Computer Vision and

541 Pattern Recognition, CVPR 2019, Long Beach, CA, USA, June 16-20, 2019. Computer

542 Vision Foundation / \{IEEE\}; 2019. p. 51-60. doi:10.1109/CVPR.2019.00014.

543 29. Madry A, Makelov A, Schmidt L, Tsipras D, Vladu A. Towards deep learning models 544 resistant to adversarial attacks. In: International Conference on Learning Representations.

545 2018. https://openreview.net/forum?id=rJzIBfZAb.

546 30. Zhang H, Yu Y, Jiao J, Xing E, Ghaoui L El, Jordan M. Theoretically principled 547 trade-off between robustness and accuracy. In: Chaudhuri K, Salakhutdinov R, editors. 548 Proceedings of the 36th International Conference on Machine Learning. Long Beach, 549 California, USA: PMLR; 2019. p. 7472-82.

550 http://proceedings.mlr.press/v97/zhang19p.html.

552 Proc 8th Int Conf Learn Represent. 2020. http://arxiv.org/abs/1905.10510. Training with Domain Adaptation. 7th Int Conf Learn Represent ICLR 2019. 2019. http://arxiv.org/abs/1810.00740. purify adversarial examples. IEEE Access. 2019;7:126582-93.

559 34. Croce F, Hein M. Reliable evaluation of adversarial robustness with an ensemble of 560 diverse parameter-free attacks. Proc 37th Int Conf Mach Learn. 2020. doi:2003.01690.

561 35. Carlini N, Wagner D. Adversarial examples are not easily detected. In: Proceedings 562 of the 10th ACM Workshop on Artificial Intelligence and Security - AISec '17. New

563 York, New York, USA: ACM Press; 2017. p. 3-14. doi:10.1145/3128572.3140444. 


\section{Tables}

566 Table 1: Targeted attack success rates $R_{s}(\%)$ of targeted UAPs against Inception V3, 567 ResNet50, and VGG16 models to each target class. $\zeta=4 \%$ for the skin lesions and chest 568 X-ray image classifications, and $\zeta=8 \%$ for the OCT image classification. The column 569 "UAP" indicates which input image set was used to generate the UAP, except for 570 "Random", which indicates random UAPs.

\begin{tabular}{|c|c|c|c|c|c|}
\hline \multirow[b]{2}{*}{ Medical images } & \multirow[b]{2}{*}{ Target class } & \multirow[b]{2}{*}{ UAP } & \multicolumn{3}{|c|}{ Model architecture } \\
\hline & & & $\begin{array}{c}\text { Inception } \\
\text { V3 }\end{array}$ & ResNet50 & VGG16 \\
\hline \multirow{8}{*}{ Skin lesion } & \multirow{4}{*}{$\mathrm{NV}$} & Training & 97.9 & 99.2 & 98.7 \\
\hline & & ImageNet & 98.8 & 96.2 & 86.6 \\
\hline & & Open Images & 99.1 & 94.5 & 86.9 \\
\hline & & Random & 64.1 & 70.2 & 73.3 \\
\hline & \multirow{4}{*}{ MEL } & Training & 97.1 & 97.7 & 97.6 \\
\hline & & ImageNet & 97.1 & 96.0 & 10.5 \\
\hline & & Open Images & 96.6 & 94.5 & 10.4 \\
\hline & & Random & 14.5 & 11.8 & 8.8 \\
\hline \multirow{8}{*}{ OCT } & \multirow{4}{*}{ NM } & Training & 98.2 & 99.4 & 98.6 \\
\hline & & ImageNet & 98.2 & 99.7 & 92.3 \\
\hline & & Open Images & 99.4 & 99.8 & 94.0 \\
\hline & & Random & 27.6 & 29.3 & 26.5 \\
\hline & \multirow{4}{*}{$\mathrm{CNV}$} & Training & 99.3 & 99.7 & 99.9 \\
\hline & & ImageNet & 99.2 & 35.5 & 98.3 \\
\hline & & Open Images & 99.3 & 48.3 & 96.2 \\
\hline & & Random & 26.5 & 26.1 & 25.4 \\
\hline \multirow{8}{*}{ Chest X-ray } & \multirow{4}{*}{ NORMAL } & Training & 99.3 & 99.3 & 99.6 \\
\hline & & ImageNet & 97.6 & 100 & 95.7 \\
\hline & & Open Images & 97.0 & 99.8 & 94.3 \\
\hline & & Random & 55.7 & 54.4 & 54.8 \\
\hline & \multirow{4}{*}{ PNEUMONIA } & Training & 97.8 & 99.1 & 99.8 \\
\hline & & ImageNet & 60.0 & 75.3 & 72.3 \\
\hline & & Open Images & 62.8 & 79.8 & 68 \\
\hline & & Random & 45.0 & 46.1 & 44.1 \\
\hline
\end{tabular}


571 Table 2: Fooling rates $R_{f}(\%)$ of nontargeted UAPs against Inception V3 models trained 572 from random initialization. $\zeta=8 \%$ for the skin lesions and chest X-ray image 573 classifications, and $\zeta=16 \%$ for the OCT image classification. The column "UAP" 574 indicates which input image set was used to generate the UAP, except for "Random", which 575 indicates random UAPs.

576

\begin{tabular}{lccc}
\hline UAP / Medical images & Skin lesion & OCT & Chest X-ray \\
\hline Training & 92.7 & 74.5 & 45.9 \\
ImageNet & 50.0 & 75.3 & 22.2 \\
Random & 7.3 & 9.9 & 0.4 \\
\hline
\end{tabular}


581 Figure 1: Vulnerability to nontargeted UAPs. Line plots of the fooling rate $R_{f}$ (\%) against 582 Inception V3 model versus perturbation magnitude $\zeta(\%)$ for the skin lesion (a), OCT (b), 583 and chest X-ray (c) image classifications. Legend label denotes the input image set used to 584 generate UAPs, except for "Random", which indicates random UAPs.

585 Figure 2: Clean images and their adversarial examples generated using nontargeted

586 UAPs from the ImageNet dataset, the against Inception V3 model for the skin lesion (a),

587 OCT (b), and chest X-ray (c) image classifications. $\zeta=4 \%$ in (a) and (c) $\zeta=8 \%$ in

588 (b). Labels beside the images are the predicted classes. The clean (original) images are

589 correctly classified into their actual labels.

$590 \quad$ Figure 3: Difference in the fooling rate $R_{f}(\%)$ of the UAPs according to model

591 architectures for skin lesions (a), OCT (b), and chest X-ray (c) image classifications. $\zeta=$ $5924 \%$ in (a) and (c) $\zeta=8 \%$ in (b). Dashed lines indicate $R_{f}(\%)$ of random UAPs

593 (random controls).

594 Figure 4: Visualization of nontargeted UAPs generated using training, ImageNet, and 595 Open Images datasets against Inception V3 models for skin lesion, OCT, and chest X-ray 596 image classifications. UAPs are visually emphasized for clarity; in particular, each UAP 597 is scaled by a maximum of 1 and minimum of 0 .

598 Figure 5: Normalized confusion matrices for Inception V3 models attacked using 599 nontargeted UAPs from training, ImageNet, Open Images datasets for skin lesions, OCT, 600 and chest X-ray image classifications.

601 Figure 6: Visualization of targeted UAPs generated using training images, ImageNet, and 602 Open Images datasets against Inception V3 models for skin lesion, OCT, and chest X-ray 603 image classifications. UAPs are visually emphasized for clarity; in particular, each UAP is 604 scaled by a maximum of 1 and minimum of 0 . 
606 Additional files

607

608 Additional file 1: Supplementary tables and figures. (DOCX)

609 
Figures
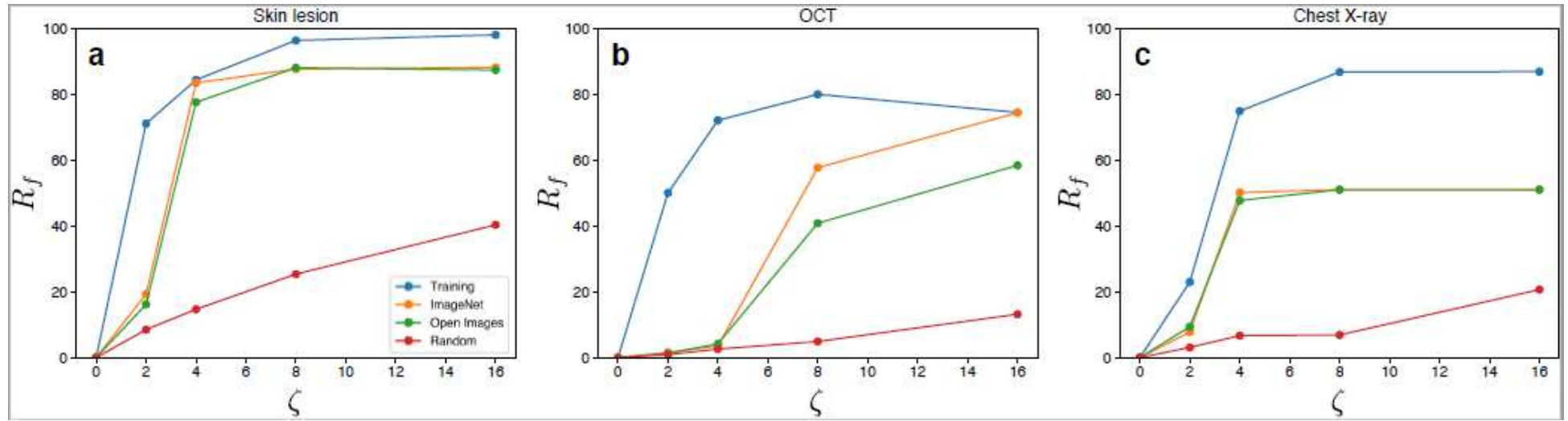

Figure 1

Vulnerability to nontargeted UAPs. Line plots of the fooling rate R_f (\%) against Inception V3 model versus perturbation magnitude $\zeta(\%)$ for the skin lesion (a), OCT (b), and chest X-ray (c) image classifications. Legend label denotes the input image set used to generate UAPs, except for "Random", which indicates random UAPs.

\section{a) Skin lesion}

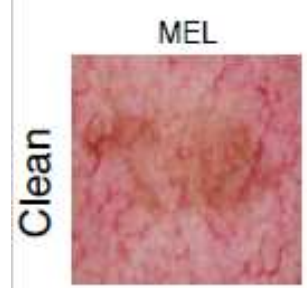

BKL

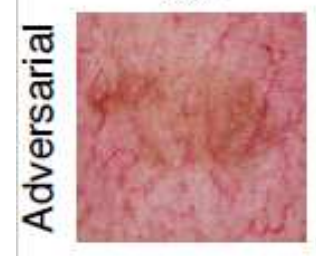

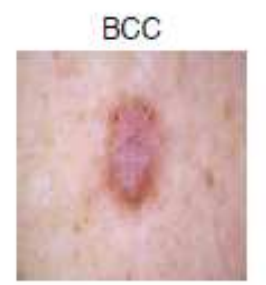

MEL
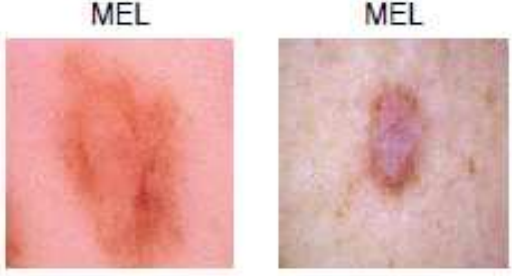

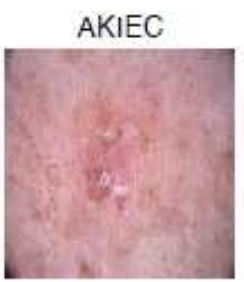

b) OCT

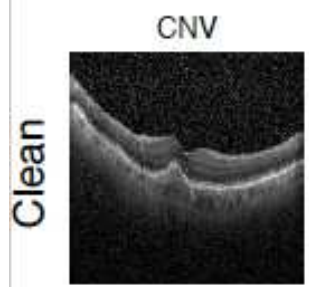

DRUSEN

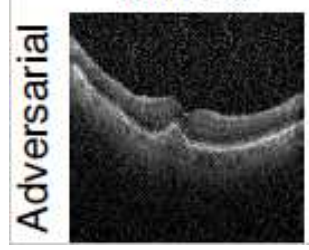

DME

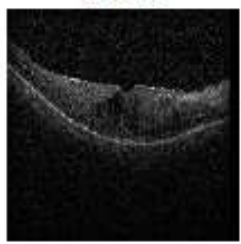

DRUSEN

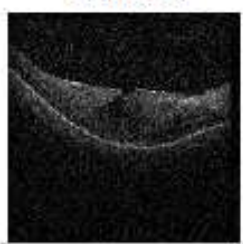

MEL

BKL

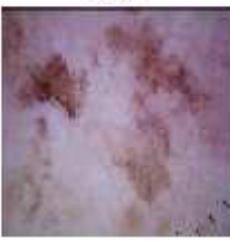

MEL
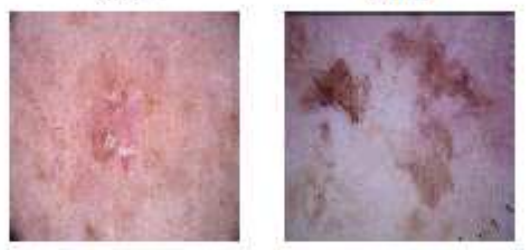

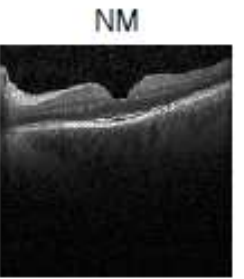

DRUSEN
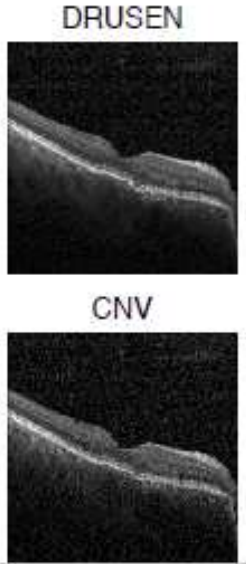

CNV

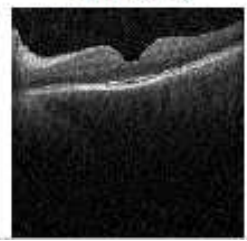

DF

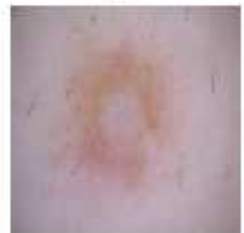

MEL

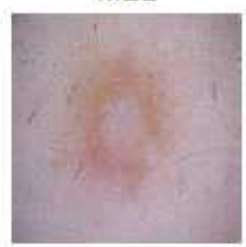

c) Chest X-ray

VASC

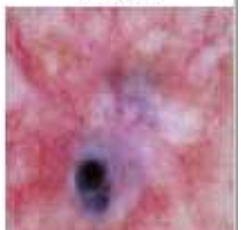

MEL

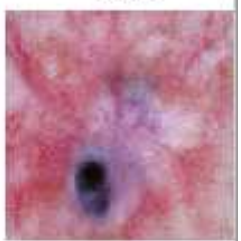

PNEUMONIA

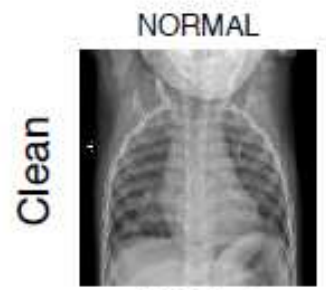

NORMAL

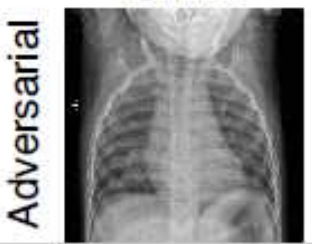

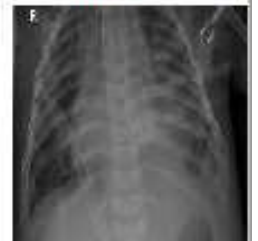

NORMAL 
Figure 2

Clean images and their adversarial examples generated using nontargeted UAPs from the ImageNet dataset, the against Inception V3 model for the skin lesion (a), OCT (b), and chest X-ray (c) image classifications. $\zeta=4 \%$ in (a) and (c) $\zeta=8 \%$ in (b). Labels beside the images are the predicted classes. The clean (original) images are correctly classified into their actual labels.
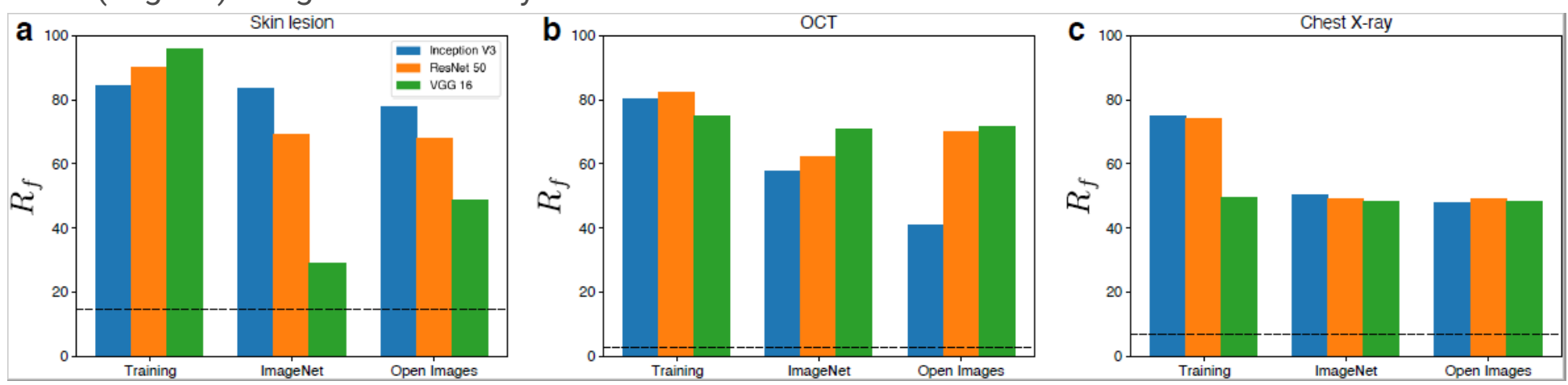

Figure 3

Difference in the fooling rate $R_{-} f(\%)$ of the UAPs according to model architectures for skin lesions (a), OCT (b), and chest X-ray (c) image classifications. $\zeta=4 \%$ in (a) and (c) $\zeta=8 \%$ in (b). Dashed lines indicate R_f (\%) of random UAPs (random controls). 


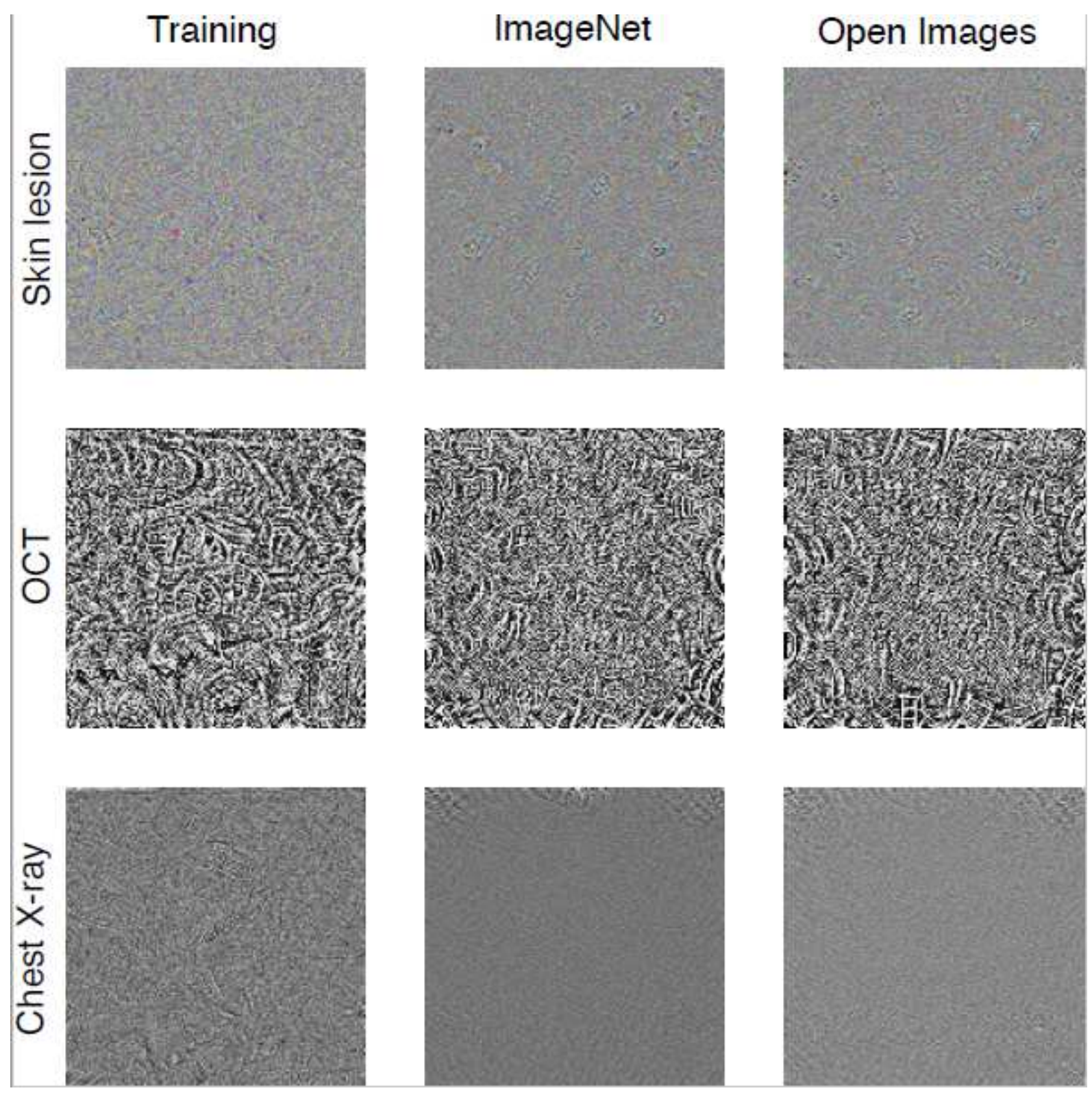

Figure 4

Visualization of nontargeted UAPs generated using training, ImageNet, and Open Images datasets against Inception V3 models for skin lesion, OCT, and chest X-ray image classifications. UAPs are visually emphasized for clarity; in particular, each UAP is scaled by a maximum of 1 and minimum of 0 . 


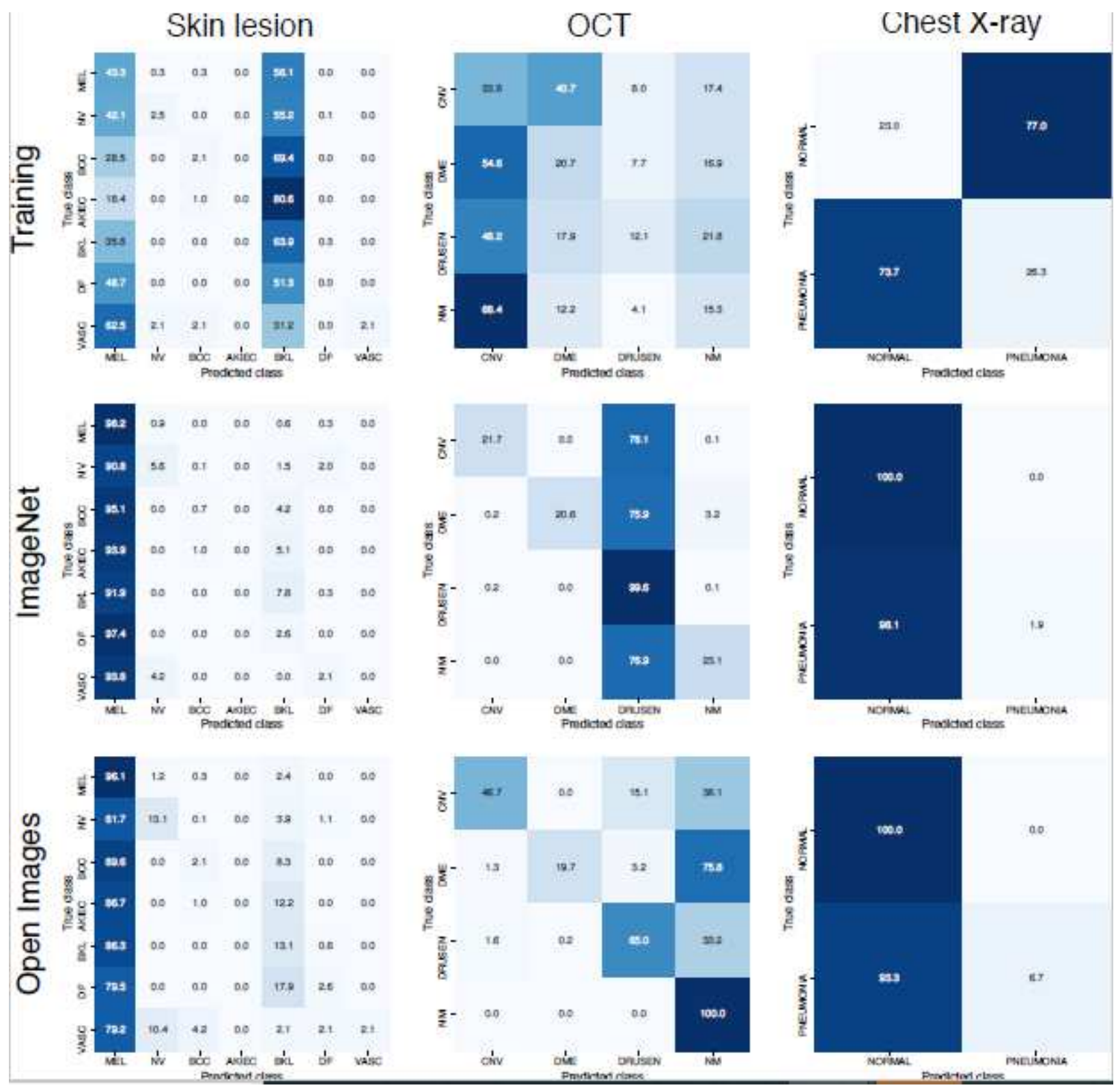

Figure 5

Normalized confusion matrices for Inception V3 models attacked using nontargeted UAPs from training, ImageNet, Open Images datasets for skin lesions, OCT, and chest X-ray image classifications. 


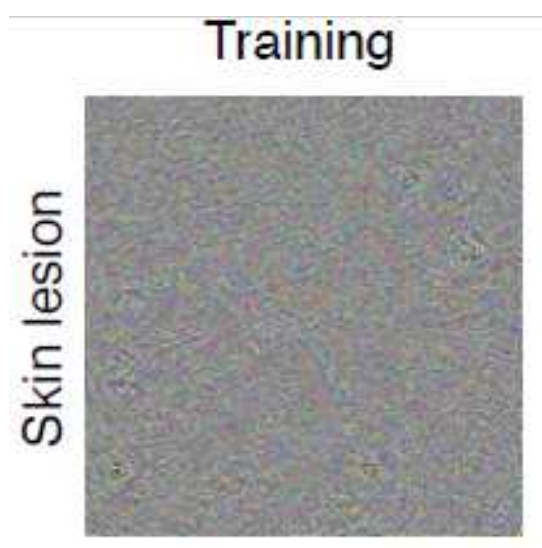

ImageNet
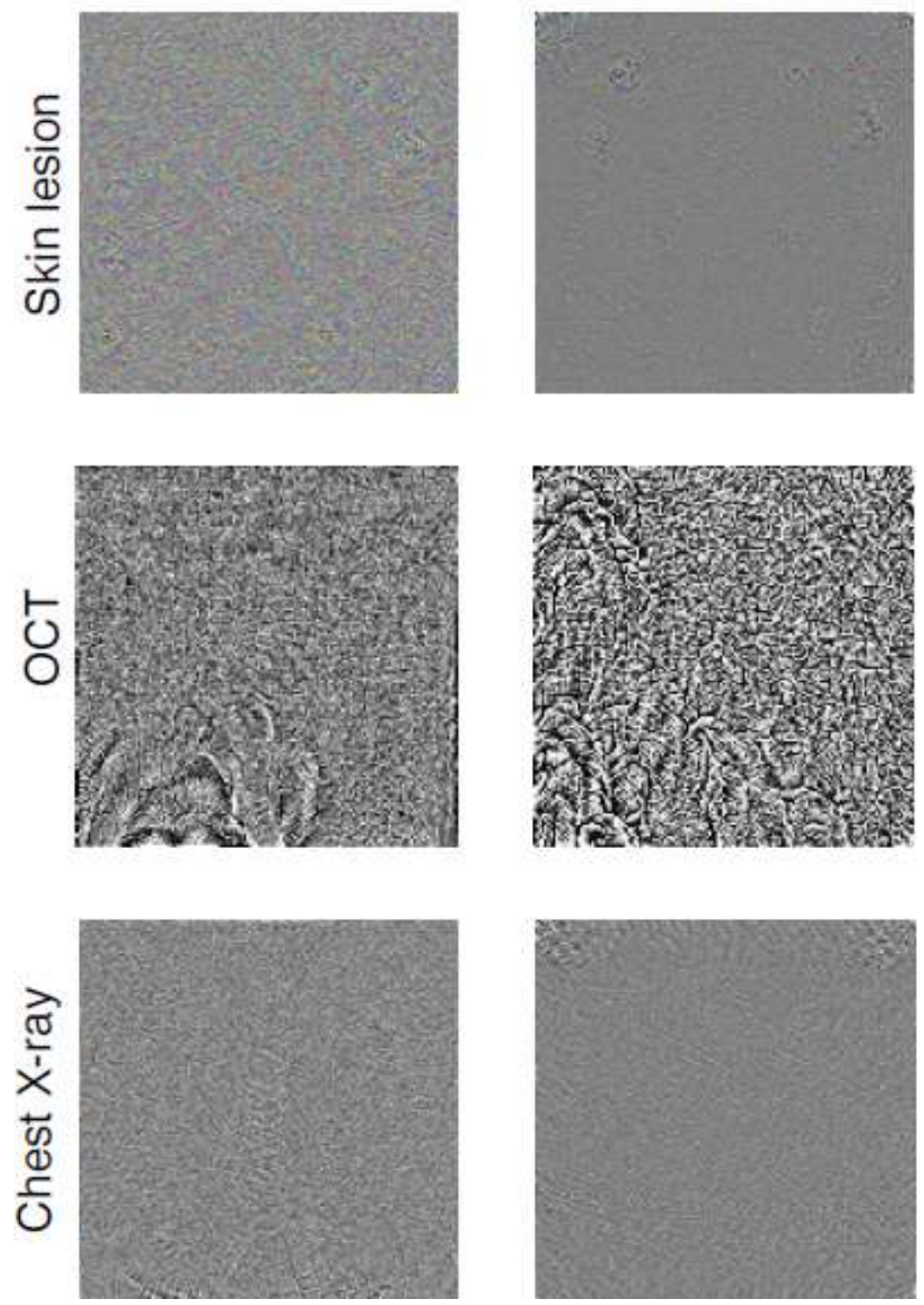

Open Images
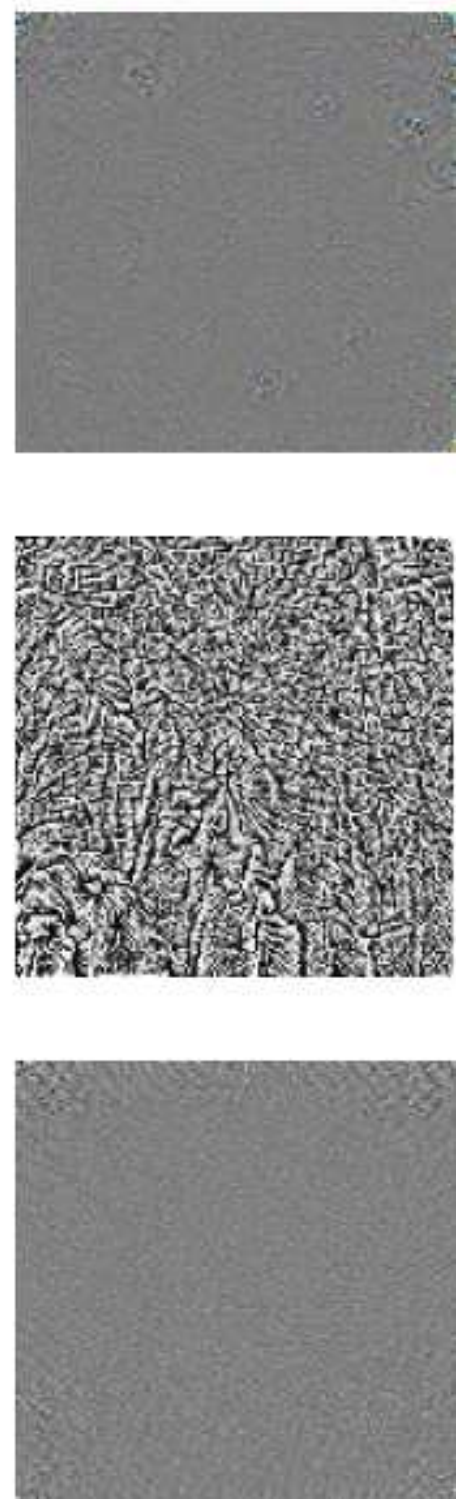

Figure 6

Visualization of targeted UAPs generated using training images, ImageNet, and Open Images datasets against Inception V3 models for skin lesion, OCT, and chest X-ray image classifications. UAPs are visually emphasized for clarity; in particular, each UAP is scaled by a maximum of 1 and minimum of 0 .

\section{Supplementary Files}

This is a list of supplementary files associated with this preprint. Click to download.

- supplementarymaterialAMHHKTNaturalUAP.docx 